\title{
Les limites de la liberté religieuse du détenu
}

\section{Laurent Mortet}

\section{(2) OpenEdition}

\section{Journals}

Édition électronique

URL : http://journals.openedition.org/rdr/1057

DOI : $10.4000 /$ rdr. 1057

ISSN : 2534-7462

Éditeur

Presses universitaires de Strasbourg

\section{Édition imprimée}

Date de publication : 10 mai 2016

Pagination : 117-128

ISBN : 978-2-86820-954-2

ISSN : 2493-8637

\section{Référence électronique}

Laurent Mortet, "Les limites de la liberté religieuse du détenu », Revue du droit des religions [En ligne],

1 | 2016, mis en ligne le 12 février 2020, consulté le 19 novembre 2020. URL : http://

journals.openedition.org/rdr/1057 ; DOI : https://doi.org/10.4000/rdr.1057

\section{(c) (7) (3)}

La revue du droit des religions est mise à disposition selon les termes de la Creative Commons Attribution - Pas d'Utilisation Commerciale 4.0 International - CC BY-NC 4.0. 


\section{LES LIMITES DE LA LLBERTÉ RELIGIEUSE DU DÉTENU}

\section{Laurent MORTET}

Docteur en droit privé et sciences criminelles de I'Université de Lorraine

\section{RÉSUMÉ}

La liberté religieuse, comme toute autre liberté fondamentale, bénéficie au détenu, dans la limite des contingences inhérentes à la détention. La protection de la liberté religieuse souffre d'abord de sa confrontation avec les impératifs liés à la sécurité et au bon ordre de l'établissement. D'autre part, dans le contexte particulier de la privation de liberté, seule la reconnaissance d'une obligation positive mise à la charge de l'État pour organiser le culte en détention est de nature à ne pas trop réduire la liberté religieuse du détenu. La protection de la liberté religieuse pourrait alors souffrir de sa confrontation avec les impératifs de neutralité du service public et de laïcité. L'obligation positive naît en jurisprudence, même si son intensité est contrastée, selon les pans de la liberté religieuse concernés.

\section{ABSTRACT}

The right to freedom of religion, as all over fundamental rights, benefits the prisoner, in the limit of inherent contingencies in custody. The protection of freedom of religion suffers first from its confrontation with the requirements due to security and the good order of the establishment. On the other hand, in the specific context of deprivation of liberty, only the recognition of a positive obligation in the responsibility of the state to organise the religious worship in prison is likely to not reduce the prisoner's religious freedom too much. The protection of freedom of religion could suffer from its confrontation with the imperatives of neutrality of the public service and secularism. The positive obligation emerges from case law, even if its intensity is contrasted, depending on the sector of religious freedom concerned. 
Selon la loi pénitentiaire, « les personnes détenues ont droit à la liberté $\checkmark$ d'opinion, de conscience et de religion. Elles peuvent exercer le culte de leur choix, selon les conditions adaptées à l'organisation des lieux, sans autres limites que celles imposées par la sécurité et le bon ordre de l'établissement $»^{1}$. La loi n'exprime ici qu'un principe désormais acquis, selon lequel les détenus conservent leurs droits et libertés fondamentaux - sauf, par définition, la liberté d'aller et venir - dans la limite des " contingences $»^{2}$, des « sujétions ${ }^{3}$ ou encore des « contraintes ${ }^{4}$ inhérentes de la détention ${ }^{5}$, la liberté religieuse ${ }^{6}$ n'échappant pas à ce sort. En substance donc, la garantie de la liberté religieuse du détenu contre les ingérences étatiques est identique à celle de la personne libre. Il ne faudrait pas non plus conclure de cet état du droit que l'étude de la liberté religieuse en prison n'intéresse pas plus que celle de toute autre liberté fondamentale appliquée dans cet environnement. D'abord, la religion et la prison ont des liens particuliers ${ }^{7}$, quant à l'origine même de la peine privative de liberté en cellule ${ }^{8}$, et ceux-ci ont justifié la permission du financement public du service d'aumônerie pénitentiaire dès l'édiction de la loi de séparation des Églises et de l'État ${ }^{9}$. Ensuite, la privation de liberté change radicalement les conditions de la garantie de la liberté religieuse concernant la personne placée sous la mainmise de l'administration pénitentiaire, au point d'impliquer de modifier les obligations applicables dans le monde libre.

D'une part, dans le contexte contraint, la garantie de la liberté fondamentale suppose la mise à la charge de l'administration d'obligations positives. Un tel raisonnement a été dégagé par la Cour européenne des droits de l'homme, ouvrant un contrôle riche et protecteur, s'agissant de la garantie de la dignité

1. Art. 26 de la loi n 2009-1436 du 24 novembre 2009 pénitentiaire.

2. Comm.EDH, 15 mai 1980, n 8317/78, McFeeley c/ Royaume-Uni.

3. CE, ord. réf., 8 sept. 2005, n 284803, Garde des Sceaux c/ Bunel, Rec. CE., p. 388.

4. Cons. const., déc. 19 nov. 2009, n² 2009-593 DC, consid. n 4.

5. V. plus généralement l'article 22 de la loi pénitentiaire.

6. Le Conseil constitutionnel a tiré du principe de laïcité que « la République [garantit] le libre exercice des cultes» (Cons. const., déc. 21 févr. 2013, n 2012-297 QPC, consid. $\mathrm{n}^{\circ}$ 5). La liberté religieuse est protégée à l'article 9 de la Convention européenne des droits de l'homme selon lequel « toute personne a droit à la liberté [...] de religion ». Le Conseil d'État a considéré la « liberté de culte» comme fondamentale (CE, 16 févr. 2004, $\mathrm{n}^{\circ}$ 264314, Ahmed B. c/ OPHLM Saint-Dizier) et l'a aussi rattachée au principe de laïcité (CE, 5 juill. 2013, n 361441, Euvre d'Assistance aux Bêtes d'Abattoirs).

7. Poncela P., « Prison et religion, je t'aime moi non plus », RSC 2015, p. 143.

8. Mabillon J., Réflexions sur les prisons des ordres religieux, 1726, Caen, G. Woinez, 1845 - Howard J., Etat des prisons, des hôpitaux et des maisons de force, 1777, Paris, Éd. de l'Atelier, 1994.

9. Le financement public des « services d'aumônerie » des « prisons » est permis par l'article 2 de la loi du 9 décembre 1905 concernant la séparation des Églises et de l'État. 
des détenus ${ }^{10}$. Concernant la liberté religieuse, le procédé n'est pas absent des dispositions du Code de procédure pénale, lesquelles mettent à la charge de l'État l'obligation positive d'organiser l'assistance spirituelle du détenu par un ministre de son culte ${ }^{11}$, mais sans envisager d'autres pans protégés par la liberté religieuse ${ }^{12}$. En conséquence, il revient souvent au juge de préciser les obligations en la matière. Cependant, si la protection de la dignité suppose tout autant l'existence d'une obligation positive mise à la charge de l'État dans le monde libre, tel n'est pas vraiment le cas pour la matière de la liberté religieuse, en raison du principe de neutralité ${ }^{13}$. S'il est une chose déjà délicate pour le juge de circonscrire le fait religieux dans le monde libre ${ }^{14}$ en défense de la laiicité ${ }^{15}$, de la neutralité ou du « vivre ensemble ${ }^{16}$, il est sûrement une chose plus délicate encore pour lui que d'organiser positivement les éléments de la liberté religieuse en détention, sans menacer les équilibres trouvés à l'extérieur. D'autre part, même dans le monde libre, l'action de l'État peut s'avérer nécessaire pour endiguer l'expression religieuse excessive, afin de protéger la liberté de conscience ou même religieuse d'autrui et assurer ainsi la neutralité d'un espace dans lequel se rencontrent croyances et convictions différentes. En prison, la religion ne saurait être trop cantonnée à l'intime, dans un monde où il en subsiste trop peu. Cependant, les nécessités pénitentiaires, celles tenant à la "sécurité et [au] bon ordre de l'établissement », à cause de la promiscuité, pourraient justifier la mise à la charge du détenu d'obligations négatives quant à sa pratique religieuse encore plus sévères que celles qui existent à l'extérieur.

C'est donc à travers la désignation des différentes obligations identifiables, celles négatives et réductrices de la liberté religieuse mises à la charge de l'administration pénitentiaire ou des détenus, comme celles positives et protectrices de la même liberté mises à la charge de l'administration pénitentiaire,

10. CEDH, Gde ch., 26 oct. 2000, n 30210/96, Kudla c/ Pologne, $\S 94$.

11. CPP, art. R. 57-9-3 et s.

12. Il est précisé de manière générique que « chaque personne détenue doit pouvoir satisfaire aux exigences de sa vie religieuse, morale ou spirituelle» (CPP, art. R. 57-9-3).

13. Celui-ci est un principe constitutionnel relié, comme la liberté de culte, au principe de laïcité (Cons. const., déc. 21 févr. 2013, n 2012-297 QPC, consid. n 5) - V. aussi CE, 16 mars 2005, n² 265560, Ministère de l'outre-mer c/ Gouvernement de Polynésie, Rec. CE, p. 168.

14. V. pour une proposition de synthèse de la jurisprudence du Conseil d'État, LaLlLEt A. et GefFray E., « Le Conseil d'État, gardien du temple : bref retour sur 25 ans de laïcité », AJDA 2014, p. 104.

15. Larticle $1^{\text {er }}$ de la Constitution dispose que « La France est une République indivisible, laïque, démocratique et sociale. »

16. CEDH, Gde ch., $1^{\text {er }}$ juill. 2014, n 43835/11, S.A.S. c/ France, $\$ 141$. 
que l'intensité des limites de la liberté religieuse du détenu peut être évaluée. Seule une certaine autonomie dans la protection de la liberté religieuse du détenu, au regard des principes applicables dans le monde libre, est de nature à empêcher que la privation de liberté n'atteigne la substance du droit fondamental. Le régime de ces obligations sera recherché dans les grands cas de limitation de la liberté religieuse du détenu, ceux liés à la sécurité et au bon ordre de l'établissement pénitentiaire, dont la vigueur se maintient (1), et ceux liés à l'insuffisance des moyens matériels à la disposition de l'administration pénitentiaire, dont la vigueur est en déclin (2).

\section{LE MAINTIEN DES LIMITATIONS LIÉES À LA SÉCURITÉ ET AU BON ORDRE DE L'ÉTABLISSEMENT PÉNITENTIAIRE}

Le pouvoir d'altérer la liberté religieuse ne saurait confiner à l'arbitraire et la réduction appliquée doit respecter la légalité, être nécessaire ${ }^{17}$ et proportionnée $^{18}$. L'examen de la situation - plutôt que du droit - pénitentiaire montre que la liberté religieuse est fortement entamée par ces considérations. D'une part, le contenu de l'assistance spirituelle du détenu isolé rappelle que les obligations positives mises à la charge de l'administration pénitentiaire peuvent être réduites par celles-ci (1.1). D'autre part, la limitation de l'espace dans lequel le détenu peut manifester ses croyances ou les interrogations soulevées autour de la fourniture des repas confessionnels attestent que des obligations négatives strictes peuvent être imposées pour réglementer le culte en détention en regard de ces mêmes considérations (1.2).

\subsection{LA RÉDUCTION DES OBLIGATIONS POSITIVES RÉGLEMENTAIRES}

La faculté pour le détenu de participer à des activités cultuelles collectives sous la direction de l'aumônier ${ }^{19}$ est suspendue pendant l'isolement à titre

17. V. pour l'interdiction de l'accès à des revues en lien avec les croyances du détenu, TA Lille,

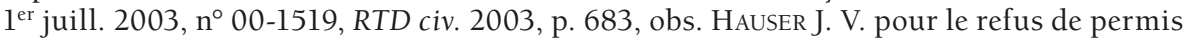
de visite du ministre du culte, CAA Bordeaux, 20 oct. 2009, n 08BX03245, AJDA 2010, p. 272, concl. FABIEN M.

18. V. pour le contrôle de l'interdiction de porter la barbe dans les établissements pénitentiaires américains confrontée à la liberté religieuse des détenus, Cour suprême des États-Unis, 20 janv. 2015, Holt v. Hobbs [574 US], RDLF 2015, chron. $n^{\circ}$ 6, comm. Hockmann T. ; ADL, 30 janv. 2015, comm. MORRI J.

19. CPP, art. R. 57-9-4 : « Les offices religieux, les réunions cultuelles et l'assistance spirituelle aux personnes détenues sont assurés, pour les différents cultes, par des aumôniers agréés. » 
disciplinaire ${ }^{20}$ ou à titre de mesure de sûretée ${ }^{21}$. Le Conseil d'État, saisi de la légalité de la suspension pour la sanction disciplinaire, au regard notamment de l'article 9 de la Convention européenne des droits de l'homme, a estimé qu'« eu égard à l'objectif d'intérêt général de protection de la sécurité et du bon ordre dans les établissements pénitentiaires qu'elles poursuivent, à la durée maximale de la sanction en cause et aux droits dont continuent à bénéficier [...] les détenus ", la suspension des activités collectives ne porte pas " une atteinte excessive au droit de ces derniers de pratiquer leur religion $»^{22}$. La suppression totale du droit à exercer le culte est donc proscrite ${ }^{23}$, comme la réduction prolongée ${ }^{24}$. Quant aux sources, d'une part, le raisonnement du juge administratif s'opère pratiquement uniquement sur les dispositions infra-législatives, montrant l'importance de la compétence réglementaire dans la délimitation de la liberté religieuse du détenu ${ }^{25}$. La validation constitutionnelle ${ }^{26} \mathrm{du}$ droit disciplinaire pénitentiaire au regard du principe de la légalité a été bien conciliante ${ }^{27}$. Quant au contrôle du juge administratif, d'autre part, l'admission de la suspension des activités de culte pour toute faute sanctionnée de la cellule disciplinaire, sans distinguer, par exemple, celles commises durant les activités cultuelles, révèle un contrôle peu exigeant.

La réduction des mêmes activités pourrait en tout cas violer la liberté religieuse en ce qui concerne l'isolement de sûreté, au regard de sa durée prolongée ${ }^{28}$, malgré les aménagements prévus ${ }^{29}$. Une telle considération n'est

20. CPP, art. R. 57-7-44: la sanction de cellule disciplinaire emporte durant toute sa durée « la suspension de l'accès aux activités».

21. CPP, art. R. 57-7-62 : si le détenu conserve « ses droits [...] à l'exercice du culte », il «ne peut participer aux promenades et activités collectives auxquelles peuvent prétendre les personnes détenues soumises au régime de détention ordinaire ».

22. CE, 11 juin 2014, n 365237, M. B., Rec. CE, tables; AJDA 2014, p. 1236, obs. BiGET C. ; D. actu., 18 juin 2014, obs. PASTOR J.-M. ; JCP A, act. n 505, obs. TouZEIL-Divina M.

23. Le détenu en cellule disciplinaire conserve le droit de s'entretenir avec l'aumônier (V. la combinaison des articles R. 57-9-6 et R. 57-9-7 CPP).

24. Le placement en cellule disciplinaire est au maximum de vingt jours pour les adultes (CPP, art. R. 57-7-47).

25. V. pour le panorama de ces sources, les visas de la note du 16 juillet 2014 relative à la pratique du culte en détention (NOR : JUSK1440001N).

26. Cons. const., déc. 19 nov. 2009, n 2009-593 DC, précit.

27. Schmitz J., « La Constitution, la loi, le règlement et l'exécution des peines de détention », RFDA 2015, p. 148.

28. V. pour la durée de l'isolement de sûreté, les articles R. 57-7-67 et s. CPP.

29. Le détenu peut, par dérogation, obtenir une «autorisation spécifique » pour participer à une activité collective et «dans toute la mesure du possible et en fonction de la personnalité de la personne détenue, des activités communes aux personnes détenues placées à l'isolement » sont organisées (CPP, art. R. 57-7-62). 
pas sans importance, alors que l'administration pénitentiaire entend combattre le radicalisme religieux en prison. D'ailleurs, une expérimentation est menée à Fresnes depuis l'automne 2014 pour regrouper certains détenus considérés comme intégristes et son extension a été annoncée. Le Contrôleur général des lieux de privation de liberté a rendu un avis défavorable sur la mesure ${ }^{30}$, soulignant le risque que celle-ci cause " grief » aux regroupés du fait de la limitation de leurs droits, ce qui apparaît somme toute vraisemblable, concernant une expérimentation, dont on connaît encore peu les effets, mais qui est présentée comme une mise à l'isolement des détenus djihadistes. Il y a sans doute ici une nouvelle illustration de l'habitude prise par l'administration pénitentiaire de gérer la sécurité et le bon ordre des établissements par la création d'un "régime spécifique de détention ${ }^{31}$ réducteur des droits fondamentaux des détenus, sans en avoir la moindre compétence ${ }^{32}$, et dans la violation du principe de la légalité.

\subsection{LA RÉGLEMENTATION DU CULTE PAR L'ÉDICTION D'OBLIGATIONS NÉGATIVES}

Il est difficile de contester qu'on ne vit pas plus « ensemble » ailleurs qu'en prison. Faut-il dès lors assurer plus encore la neutralité de l'espace commun -à défaut d'espace public - pour protéger le "vivre ensemble ", ou au contraire admettre plus largement l'expression religieuse dans celui-ci, en compensation de la réduction de la sphère intime et privée inhérente à la détention ? La réglementation du culte en prison défend fermement la première position. L'expression religieuse est cantonnée dans des lieux privatifs, soit la cellule à titre individuel, soit les locaux affectés à cet usage à titre collectif ${ }^{33}$, sans que les textes ne semblent avoir clairement envisagé le cas de la cellule collective. De même, le port ostentatoire de signes religieux est interdit dans les parties communes des établissements pénitentiaires, au regard du règlement

30. France. Contrôleur GÉnÉral des lieux de PRivation de liberté, Avis du 11 juin 2015 sur la prise en charge de la radicalisation islamiste en milieu carcéral, JO 30 juin 2015.

31. V. pour la mesure des rotations de sécurité, qualifiée de « régime de détention spécifique », CE, ass., 14 déc. 2007, n 306432, M. Payet, Rec. CE, p. 498.

32. V. pour l'annulation de la note du Garde des sceaux instituant les rotations de sécurité pour incompétence, CE, 29 févr. 2008, n³08145.

33. Les offices « sont organisés dans un local déterminé par le chef d'établissement » (CPP, art. R. 57-9-5). Larticle 18 du règlement intérieur type des établissements pénitentiaires (V. CPP, art. R-57-6-18) prévoit que « chaque personne détenue peut exercer le culte de son choix, à titre individuel dans sa cellule ou collectivement dans les salles prévues à cet effet, en présence des intervenants d'aumônerie ». 
intérieur type ${ }^{34}$ ou de la... dernière circulaire ${ }^{35}$. Concernant le développement de pratiques de prières collectives, non pas dans la rue, mais dans la cour de promenade, le juge administratif a conforté cette approche, validant leur interdiction prise par l'autorité pénitentiaire, sur le fondement de la liberté de conscience d'une part et sur le fondement de la sécurité pénitentiaire d'autre part, sans procéder, pour le dernier point, à une démonstration vraiment poussée de sa mise en péril ${ }^{36}$.

À appliquer une forte conception de la neutralité dans la « prison républicaine »- on aimerait qu'elle le soit toujours, notamment quant à la dignité des conditions matérielles de détention - le juge ne pourrait donc mettre à la charge de l'administration aucune obligation positive pour faciliter la liberté religieuse, en dehors de l'assistance spirituelle, prévue par les textes. Un tribunal administratif a directement écarté ce raisonnement, en donnant injonction au service pénitentiaire de fournir régulièrement des repas respectant le rite halal, notant que les détenus « sont des usagers de service public dans une situation contrainte ${ }^{37}$. L'analyse est juste, pour réinterpréter le principe de laïcité dans le contexte pénitentiaire. Si le service public de restauration dans le monde libre - par exemple la cantine scolaire - peut opposer le principe de neutralité aux revendications des usagers exigeant l'obtention de repas confessionnels ${ }^{38}$ qu'ils peuvent obtenir ailleurs, le détenu demeure un usager particulier car dépendant, si bien que le seul dégagement d'une obligation positive mise à la charge de l'administration pénitentiaire de fournir des repas confessionnels lui garantit l'exercice de sa liberté religieuse. Le Conseil d'État avait pourtant soulevé, en suspendant ${ }^{39}$ l'injonction faite par le juge de premier degré, que les moyens contre sa légalité de «l'atteinte au principe de laïcité et de l'incompatibilité de la mesure ordonnée avec les exigences de la détention apparaissent,

34. Ibid. : " Le port des vêtements religieux est interdit dans les lieux à usage collectif, à l'exception de la salle de culte. Les vêtements et objets de culte doivent être transportés dans un sac de la cellule à la salle de culte ».

35. Note du 16 juill. 2014, précit, p. 10 : « Le port de signes par lesquels les personnes détenues manifestent ostensiblement une appartenance religieuse est interdit». La formule est proche de l'interdiction posée en milieu scolaire (V. l'art. $1^{\text {er }}$ de la loi $n^{\circ} 2004-228$ du 15 mars 2004).

36. CAA Bordeaux, 8 sept. 2009, AJP 2009, p. 459, obs. Herzog-Evans M. ; JCP A 2010, $\mathrm{n}^{\circ} 2207$, chron. Pacteau B.

37. TA Grenoble, 7 nov. 2013, JurisData n 2013-028112 ; JCP A, act. n 966, obs. MANDin N.

38. Kimmel-Alcover A, « Restauration scolaire et laïcité : quand la religion de l'élève s'invite à la table de la cantine », RD sanit. soc. 2014, p. 146.

39. CJA, art. R. 811-17. 
en l'état de l'instruction, comme sérieux ${ }^{40}$. Au fond ${ }^{41}$, le Conseil d'État a estimé que les dispositions réglementaires qui « sans [...] imposer à l'administration de garantir, en toute circonstance, une alimentation respectant ces convictions, ne peuvent être regardées, eu égard à l'objectif d'intérêt général du maintien du bon ordre des établissements pénitentiaires et aux contraintes matérielles propres à la gestion de ces établissements, comme portant une atteinte excessive au droit de ces derniers de pratiquer leur religion ».

À première vue, on pourrait penser que les considérations pour le «vivre ensemble » ont motivé la référence faite par le juge administratif à « l'objectif d'intérêt général du maintien du bon ordre des établissements pénitentiaires ». Mais le Conseil d'État n'a pas évoqué l'autre cause de limitation de la liberté religieuse de l'article 22 de la loi pénitentiaire, plus explicite, à savoir la « sécurité ». Par rapport à l'arrêt précédent, le Conseil d'État a même abandonné toute référence à la laïcité. Il n'a pas plus fait référence ici à la neutralité, et la liberté de conscience ne figure qu'à travers la citation des textes internationaux qui constituent les fondements de la liberté de religion en même temps que ceux de la liberté de conscience ${ }^{42}$. L'arrêt ne dénie pas non plus totalement l'existence d'une obligation positive. Car à le lire, l'administration pénitentiaire doit bien garantir dans certaines circonstances la fourniture de repas confessionnels, ce qui est déjà plus protecteur que les timides dispositions du règlement intérieur ${ }^{43}$. Dès lors, il nous semble que "le bon ordre » vise plutôt la désorganisation qu'aurait causée l'obligation faite à l'administration pénitentiaire de devoir fournir régulièrement des menus halal dans l'ensemble des prisons françaises, l'argument rejoignant celui de l'insuffisance des moyens matériels, autre cause de limitation de la liberté religieuse, plus nettement en déclin que les considérations sécuritaires.

40. CE, 16 juill. 2014, no 377145, Garde des Sceaux, Min. de la justice cl. M. B., AJDA 2014, p. 2321, comm. PrÉlOt P.-H.

41. CE, 25 févr. 2015, n 375724, X c/ Secrétariat général du gouvernement, AJDA 2015, p. 421, obs. PASTOR J.-M. ; JCP A 2015, act. n² 243, obs. LANGELIER É. : il était saisi, dans une affaire différente, de la légalité du refus d'abroger l'art. R. 57-6-18 CPP.

42. Conv. EDH 4 nov. 1950, art. 9 et PIDCP, art. 18.

43. Art. 9 du règlement intérieur type : l'administration pénitentiaire doit fournir des repas confessionnels " dans toute la mesure du possible». 


\section{LE DÉCLIN DES LIMITATIONS LIÉES À L'INSUFFISANCE DES MOYENS MATÉRIELS DE L'ADMINISTRATION PÉNITENTIAIRE}

Le monde pénitentiaire n'est pas seulement obsédé par la sécurité, il souffre aussi de l'insuffisance de moyens matériels l'empêchant de garantir ne serait-ce que des conditions matérielles de détention dignes. En cette matière pourtant, l'insuffisance des moyens n'empêche pas le constat par la Cour européenne des droits de l'homme d'un traitement inhumain et dégradant ${ }^{44}$. En revanche, au regard de la jurisprudence administrative récente, la protection de la liberté religieuse en détention n'est pas encore indifférente à cette cause de limitation. Heureusement, sa vigueur est en déclin, comme le montrent, pour le cas de la fourniture du repas confessionnel, le dégagement principalement jurisprudentiel d'une obligation positive de moyens atténuée à la charge de l'administration (2.1), et plus encore, pour la fourniture de l'assistance spirituelle, le refus du juge d'atténuer l'obligation positive réglementaire (2.2).

\subsection{LE DÉGAGEMENT JURISPRUDENTIEL D'UNE OBLIGATION POSITIVE DE MOYENS ATTÉNUÉE}

La Cour européenne des droits de l'homme, saisie de la nature de l'obligation de l'administration pénitentiaire à fournir des repas conformes aux croyances religieuses $^{45}$, a estimé, concernant une personne se réclamant du bouddhisme, que l'État devait lui fournir des repas sans préparation animale, conformément à ses croyances ${ }^{46}$. La Cour a admis cependant que le droit du détenu de bénéficier d'un repas confessionnel puisse être limité par l'importance des incidences financières que son respect entraînerait ${ }^{47}$. Tel n'était pas le cas en l'espèce, puisque l'accommodement des repas aux convictions du détenu supposait uniquement de retrancher un aliment, sans imposer aucune autre transformation ou utilisation de produits spéciaux ${ }^{48}$ : l'obligation positive de moyens ainsi posée était très atténuée. Comme nous l'avons déjà évoqué, le juge administratif français a été aussi saisi de la question. Un tribunal administratif a largement dépassé l'exigence européenne, pour

44. CEDH, 29 avr. 2003, n 38812/97, Poltoratski c/ Ukraine, \$148.

45. CEDH, 7 déc. 2010, $\mathrm{n}^{\circ}$ 18429/06, Jakobski c/ Pologne. V. pour un cas et une analyse similaires, CEDH, 17 déc. 2013, $n^{\circ}$ 14150/08, Vartic c/ Roumanie ( $\left.n^{\circ} 2\right)$.

46. CEDH, 7 déc. 2010, Jakobski, précit., §48 et s.

47. Ibid., $\$ 50$.

48. Ibid., $\$ 59$. 
formuler l'injonction à l'administration pénitentiaire de fournir « régulièrement » des menus composés de viande halal au détenu. L'injonction avait été suspendue par le Conseil d'État ${ }^{49}$, juste à temps ${ }^{50}$. Celui-ci retenait une analyse radicalement différente de celle du tribunal administratif qui avait longuement minoré les incidences matérielles de la fourniture de repas halal et estimé la demande « raisonnable ${ }^{51}$ : le Conseil d'État affirmait que « la distribution au sein du centre pénitentiaire [...] de repas composés de viande "halal" imposerait des travaux d'un montant très élevé et matériellement difficiles à réaliser ou, à supposer l'approvisionnement par un sous-traitant matériellement possible, des coûts qui demeureraient élevés ${ }^{52}$. Une telle différence d'appréciation aurait mérité sans doute plus d'explications de ce dernier, qui s'est fondé sur des éléments financiers fournis par l'administration pénitentiaire, sans les restituer.

Sans surprise, le juge d'appel avait, ultérieurement à la suspension, annulé le jugement ${ }^{53}$, au motif que «l'administration pénitentiaire ménage [...] un juste équilibre entre les nécessités du service public et les droits des personnes détenues en matière religieuse ", par le fait que celle-ci propose, en plus du menu normal, des menus de substitution, des menus halal lors des fêtes religieuses et des produits halal à l'achat. Le Conseil d'État, saisi dans une autre affaire au fond, a semblé préserver les apports de l'arrêt de la cour administrative d'appel, pour ne pas imposer la fourniture de repas halal « en toute circonstance ${ }^{54}$, sans préciser dans quelles circonstances cela s'avérait nécessaire. De cet enchaînement, il résulte bien une obligation positive de l'administration pénitentiaire à fournir au détenu une alimentation conforme à ses croyances, même si celle-ci est largement atténuée, pour n'être complète qu'aux moments des fêtes religieuses, et dont, dans ces conditions, on se réjouira peu qu'elle dépasse en l'état le standard européen. Il y a ici un désaccord important entre le juge administratif et le Contrôleur général des lieux de la privation de liberté - lequel avait longuement plaidé pour la fourniture de repas confessionnels ${ }^{55}$ _

49. CE, 16 juill. 2014, nº 377145 , précit.

50. Quelques jours plus tard, la cour administrative d'appel de Lyon devait se prononcer en appel, après avoir déjà refusé de suspendre l'injonction (CAA Lyon, 20 mars 2014, $\mathrm{n}^{\circ}$ 14LY00115).

51. TA Grenoble, 7 nov. 2013, précit.

52. CE, 16 juill. 2014, n 377145 , précit.

53. CAA Lyon, 22 juill. 2014, nº 14LY00113, AJDA 2014, p. 2321 ; ibid., p. 1524, obs. MONTECLER M.-Ch DE ; JCP A 2014, n 2323, obs. Nicoud F.

54. CE, 25 févr. 2015, n 375724, X c/ Secrétariat général du gouvernement, précit.

55. France. Contrôleur GÉnÉral des lieuX de Privation de liberté, Rapport annuel 2013, p. 247 et $s$. 
qui surprend après la belle entente entrevue sur la question des conditions matérielles de détention de la prison des Baumettes ${ }^{56}$.

\subsection{LE REJET DE L'ATTÉNUATION DE L'OBLIGATION POSITIVE RÉGLEMENTAIRE}

L'apport récent le plus protecteur de la liberté religieuse du détenu réside dans la reconnaissance du droit du Témoin de Jéhovah en prison de bénéficier de l'assistance spirituelle dirigée par un ministre du culte, et ce dans un certain consensus $^{57}$. La jurisprudence administrative a ainsi appliqué le principe selon lequel la substance de la liberté religieuse protégée est identique en prison et dans le monde libre, tirant les conséquences que, dans cet espace, le caractère cultuel du mouvement des Témoins de Jéhovah avait fait l'objet d'une certaine reconnaissance ${ }^{58}$. Le juge administratif a surtout tranché la question de l'application de l'assistance spirituelle aux cultes minoritaires, contestée par l'administration pénitentiaire. Adoptant la solution du rapporteur public, qui s'était appuyé sur l'existence d'une « obligation renforcée d'assurer la liberté de religion en prison ${ }^{59}$, le juge administratif a donc jugé « que l'insuffisance du nombre de détenus se revendiquant de la confession [d'un culte] ne pouvait constituer un motif de nature à justifier un refus de délivrer un agrément en qualité d'aumônier ${ }^{60}$. Le Conseil d'État avait même développé un principe plus large selon lequel l'administration pénitentiaire « doit [...], dans la mesure où les locaux le permettent et dans les seules limites du bon ordre et de la sécurité, permettre l'organisation du culte dans les établissements ${ }^{61}$, obligation positive reposant directement sur les fondements constitutionnels de la laïcité ${ }^{62}$, sans qu'elle n'ait empêché le même juge d'atténuer plus tard

56. CE, ord. réf. 22 déc. 2012, n 364584, Section française de l'Observatoire international des prisons.

57. CE, 16 oct. 2013, n' 351115, Garde des Sceaux, ministre de la Justice c/ M. Fuentes, AJDA 2013, p. 2386, concl. Hédary D. ; JCP A 2013, act. n 861, obs. Touzeil-Divina M. ; D. 2014, p. 14, chron. Dieu F. - CAA Paris, 30 mai 2011, n 10PA03589, AJDA 2011, p. 2185, obs. Montecler M.-Ch. De et comm. Vidal Delplanque P. - CAA Paris, 27 juin 2011, $\mathrm{n}^{\circ}$ 10PA3749, AJDA 2011, p. 2121, comm. SiRinelli M. - TA Paris, 6 juill. 2007, n 0613450 , AJDA 2007, p. 2097, concl. CÉlÉRIÉ T. - HALDE, délib. n² 2010-43, 22 févr. 2010, AJP 2010, p. 455 , obs. Herzog-Evans M.

58. CE, 23 juin 2000, $\mathrm{n}^{\circ}$ 215109, Min. éco., fin. et ind. c/ Assoc. locale pour le culte des témoins de Jéhovah de Clamecy - CEDH, 30 juin 2011, n 8916/05, Assoc. Les Témoins de Jéhovah c/ France.

59. HÉDARY D., précit.

60. CE, 16 oct. 2013, n³51115, précit.

61. Ibid.

62. Const. 4 oct. 1958, art. $1^{\text {er }}$ et Déclaration des droits de l'homme et du citoyen, art. 10. 
l'intensité d'une telle obligation pour la fourniture des repas halal. Finalement, si la fameuse loi de séparation des Églises et de l'État de 1905 avait reconnu la spécificité de l'exercice du culte en milieu pénitentiaire, la situation n’a guère évolué pour le détenu, dont la meilleure garantie de la liberté religieuse réside toujours dans l'aumônerie. 